\title{
Acerca de la patologización y medicalización de los malestares infantiles actuales y sus derechos en juego
}

\author{
About the pathologization and medicalization \\ of current childbood discomforts and their rights \\ at stake
}

Sobre a patologização e medicalização das doenças infantis atuais e seus direitos "em jogo" $\grave{A}$ propos de la pathologisation et de la médicalisation du mal être des enfants et de la garantie de leurs droits

关于当前儿童时期的不适及其 “利害做关”的权利的病态化 和医学化

Gabriela Dueñas ${ }^{1}$ | Universidad del Salvador

Revista Derechos en Acción ISSN 2525-1678/ e-ISSN 2525-1686

Año 4/Nº 12 Invierno 2019 (21 junio a 20 septiembre), 178-189

DOI: https://doi.org/10.24215/25251678e304

ORCID: https://orcid.org/0000-000

Recibido: $19 / 05 / 2019$

Aprobado: 01/07/2019

Resumen: El trabajo aborda el fenómeno caracterizado por una tendencia en franco incremento a "patologizar y medicalizar" los malestares psicosociales actuales, en especial los infantiles. Atentos a sus alcances e implicancias, advierten a la vez que el problema resulta aún más grave cuando avanza sobre la niñez y la adolescencia, mientras nos convocan a preguntarnos, si el mismo no debiera ser considerado como una suerte de violencia simbólica, pero también real, que sociedades como la nuestra ejercen en estos tiempos sobre niños, niñas y adolescentes que se encuentran en situación de mayor vulnerabilidad psicosocial, teniendo

1 Doctora en Psicología. Licenciada en Educación. Psicopedagoga. 
en cuenta que, impulsados por las lógicas del Mercado de la industria farmacéutica. Realiza su propuesta desde una perspectiva que imponga una reflexión crítica que historice la intervención profesional, revirtiendo la cultura mercantilista que encierra, silencia y excluye a nuestros niños niñas y adolescentes, de modo de poder construir colectivamente dispositivos inclusivos.

Palabras claves: Infancia-medicalización- derechos

Abstract: The present work addresses the phenomenon characterized by a tendency to increase "pathologize and medicalize" the current psychosocial discomforts, especially children. Its scope and implications are reflected, while the problem is even more serious when it advances on childhood and adolescence.At the same time, we wonder if it should not be considered as a kind of symbolic violence, but also real, that societies such as ours exercise in these times on children and adolescents who are in a situation of greater psychosocial vulnerability driven by Logic of the pharmaceutical industry market. A critical reflection is proposed that historicizes professional intervention, reversing the mercantilist culture that encloses, silences and excludes our children and adolescents, so that we can collectively build inclusive devices.

Keywords: Childhood-medicalization- rights

Resumo: 0 trabalho aborda o fenômeno caracterizado por uma tendência em franco aumento a "patologizar e medicalizar" os desconfortos psicossociais atuais, especialmente os infantes. Atentos ao seu alcance e implicações, advertem ao mesmo tempo que o problema é ainda mais grave quando avança sobre infância e adolescência, enquanto nos convocam a perguntar-nos, se não deve ser considerado como um tipo de violência simbólica, mas também real, que sociedades como a nossa exercem nestes tempos sobre crianças e adolescentes que estão em uma situação de maior vulnerabilidade psicossocial, tendo em conta que, impulsionado pela lógica do mercado da indústria farmacêutica. Faça sua proposta desde uma perspectiva que imponha uma reflexão crítica que historie a intervenção profissional, revertendo a cultura mercantilista que encerra, silencia e exclui nossas crianças e adolescentes, para que possamos construir coletivamente dispositivos inclusivos.

Palavras-chave: Infância-medicalização-direitos. 
Résumé: Le présent travail aborde le phénomène caractérisé par une tendance à pathologiser et médicaliser les mal-êtres psychosociaux actuels, en particulier chez les enfants.II attire l'attention sur sa portée et ses implications, les conséquences pouvant être plus grave encore quand il s'agit de l'enfance et de l'adolescence. II nous invite à nous demander si ceci ne devrait pas être considéré comme une violence symbolique, mais aussi réelle, exercée par la société sur les enfants et adolescents en situation de vulnérabilité psychosociale accrue, impulsée par la logique de marché de l'industrie pharmaceutique. II propose une réflexion critique en historisant l'intervention des professionnels de la santé et mettant à jour ainsi cette culture mercantiliste qui enferme, réduit au silence et exclut nos enfants et nos adolescents, afin que nous puissions construire collectivement des dispositifs inclusifs.

Mot-clés: Enfance - médicalisation - droits

摘要: 这项工作涉及这种现象, 其特征是倾向于增加“病理化和医学 化” 当前心理社会不适,特别是儿童. 关注其范围和影响, 他们同时警 告说，当儿童和青少年进步时问题更加严重，同时召唤我们扪心自 问，是否应该将其视为一种象征性的暴力, 而且也是真实的我们这 样的社会在这些时期对处于心理社会脆弱程度较高的儿童和青少 年进行锻炼, 同时考虑到制药业市场的逻辑推动. 从一个批判性反 思的角度提出你的建议, 这种反思将专业干预历史化, 扭转了包围, 默和排斥我们的儿童和青少年的重商主义文化, 以便我们能够共同 建立包容性的设备.

关键字: 关键字: 童年, 医疗, 权利

\section{A modo introductorio ${ }^{2}$}

Resulta oportuno comenzar este artículo advirtiendo que, en estos tiempos actuales y en el marco de una sociedad globalizada como la que nos toca vivir, nuestro país transita un escenario plagado de contradicciones que complejizan la discusión

\footnotetext{
2 Este trabajo abreva en un artículo de Dueñas, Gorbacz y Rattagan (2014) "La Clínica en los límites de la Ley", publicado en la Revista Generaciones N ${ }^{0} 3$ de la Facultad de Psicología de la UBA. Bs As. Editorial EUDEBA.
} 
por una salud mental más justa, menos atada al mercado, y con el eje puesto en la salud como derecho.

Desde esta perspectiva, se observa con gran preocupación cómo mientras no pocos profesionales de la salud mental y otros sectores afines, entre ellos, diversos colectivos que en nuestro país se encuentran abocados a promover los derechos de las infancias y adolescencias, denuncian como la industria farmacéutica incita el consumo de drogas psicoactivas en niños y adolescentes, no pocas corporaciones ligadas al "poder médico psiquiátrico hegemónico" insisten en cuestionar y obstaculizar la plena implementación de la legislación nacional vigente en materia de Salud, Salud Mental y Adicciones con el auspicio, como siempre, de importantes laboratorios multinacionales de psicofármacos.

Al respecto, resulta interesante observar en relación a esta temática, que en estos momentos, en que en Argentina se trata la cuestión del narcotráfico como un tema de primerísimo interés en la agenda mediática, y al mismo tiempo (¿o como consecuencia?) en la agenda social, existen más de 3 millones de argentinos que diariamente consumen psicofármacos, y entre ellos, y esto no es un "asunto menor", cada vez más niños, niñas y adolescentes.

Es decir, nos aterrorizamos del supuesto aumento del consumo de sustancias ilegales, mientras invisibilizamos el enorme consumo de sustancias psicoactivas legales. La pregunta es: ¿por qué esa disparidad abismal para medir la dimensión de uno y otro problema? ${ }^{3}$.

Sin dudas que la respuesta a este contrasentido no la debemos buscar en el plano sanitario ${ }^{4}$. Resulta oportuno sin embargo,

3 "Una mirada específica sobre la problemática del consumo de psicofármacos en Argentina 2012", SEDRONAR: en Argentina unas 3.303.629 personas (el 18\% del total de población nacional), de entre 12 a 65 años hicieron uso de tranquilizantes o ansiolíticos. Los más utilizados para calmar nervios o para poder dormir son valium, lexotanil, alplax u otros. El consumo es mayor entre las mujeres y aumenta a partir de los 35 años".

4 Es decir que en un extremo de esta historia, que termina en la patologización y medicalización de las infancias y adolescencias, y en general de la vida cotidiana de todos nosotros, y en 
señalar que son numerosos los estudios que desde el campo de la salud y otros afines, vienen hace ya tiempo refiriendo con preocupación a este fenómeno como una tendencia en franco incremento a "patologizar y medicalizar" los malestares psicosociales actuales. Atentos a sus alcances e implicancias, advierten a la vez que el problema resulta aún más grave cuando avanza sobre la niñez y la adolescencia, mientras nos convocan a preguntarnos, si el mismo no debiera ser considerado como una suerte de violencia simbólica, pero también real, que sociedades como la nuestra ejercen en estos tiempos sobre niños, niñas y adolescentes que se encuentran en situación de mayor vulnerabilidad psicosocial, teniendo en cuenta que, impulsados por las lógicas del Mercado de la industria farmacéutica, el tipo de prácticas médicas que por esta vía se promueven sobre ellos -y "por su propio Bien"- suponen un "abuso de poder" que los toma como "objeto de tratamientos", mientras se silencia su sufrimiento apelando a dispositivos químicos y de disciplinamiento que vulneran, entre otros, su derecho a "ser escuchados".

\section{II. ¿A qué referimos con "patologización y medicalización" de los malestares de la vida y de las infancias actuales?}

Antes de avanzar sobre esta cuestión, resulta conveniente realizar la siguiente aclaración. Con estos términos, no se está cuestionando de manera generalizada el avance de los conocimientos médicos que posibilita la detección temprana de una gama cada vez más amplia de enfermedades, ni los tratamientos médicos a base de medicación, menos aún el desarrollo científico tecnológico de la industria farmacéutica gracias a la cual no sólo se ha logrado mejorar de modo significativo la calidad

\footnotetext{
el otro extremo, nos encontramos con el fenómeno de la formación de profesionales fuertemente atada al financiamiento de laboratorios, y por tanto, a sus intereses comerciales, y una política de drogas -que de a poco, afortunadamente vamos cuestionando y abandonando- que criminaliza el consumo de determinadas sustancias, promueve guerras sangrientas contra su comercialización, e ignora el uso indiscriminado -recetado y no recetado- de psicofármacos de curso legal
} 
de vida de amplios sectores de la población mundial que tienen acceso a ella, sino que, además, ha permitido aumentar sus expectativa de vida.

Sería una actitud necia negar estos progresos.

En el campo específico de la salud mental por ejemplo, la llegada de los psicofármacos marcó un antes y un después incuestionable.

Lo que en su lugar aquí se considera necesario poner en cuestión con el concepto de "medicalización" es una tendencia que, apoyada en concepciones de fuerte sesgo biologicista e innatista, en los últimos tiempos parece avanzar con intenciones hegemonizantes, no sólo ya sobre la población en general, sino ahora también sobre la niñez y adolescencia, reduciendo para esto de manera científicamente injustificada, complejas problemáticas socio-familiares y escolares a la idea de que todas ellas se tratan en realidad de "supuestas" deficiencias o trastornos neurocognitivos de etiología genética portados por los niñxs. Y que por esta razón, la única solución posible que se les propone entonces, parece limitarse a tratamientos médicos, centrados en la administración de drogas psicoactivas, que se acompañan a la vez de toda una serie de procedimientos complementarios de "adiestramiento conductual", que alcanzan incluso el campo de lo escolar, sin que se consideren en relación a este tipo de intervenciones -y entre otras cuestiones- sus marcados efectos "estigmatizantes".

Ahondando en la cuestión, observamos en relación a este tema que desde que el Manual de estadística descriptiva de Trastornos Mentales conocido como DSM se convirtió en la principal referencia considerada "científica" para clasificar las problemáticas y los padecimientos psíquicos de las personas,

5 Sino en el político: el país más poderoso del mundo se beneficia con las patentes de los medicamentos, con la venta de armas para la guerra contra las drogas y, de paso, construye un argumento con consenso internacional que le permite intervenir en asuntos internos de otros países. Al respecto, el ex Secretario de Seguridad de la Nación Dr. Sergio Berni señalaba en una nota que le realizaron en 2014 que EEUU es un país que " “importa las drogas y exporta las muertes" TELAM 6 de mayo de 2014. 
no pocos profesionales del campo de la salud mental parecen haber quedado sometidos a un doble imperativo biológico y de seguridad, de modo que -regidos por estas lógicas- su principal objetivo parece orientado en los últimos años sólo a detectar y perseguir la anomalía psíquica de la misma manera en que se detecta una enfermedad orgánica. De esta manera, y a modo de ejemplo paradigmático del fenómeno de la patologización y medicalización de las infancias actuales, vemos como se suelen tratar como "enfermos", rotulados de "ADD-H", a no pocos niños, niñas y adolescentes que se rebelan contra el sistema escolar, y a los que se les suministra Ritalina para lograr disciplinarlos, mientras se cierran los ojos ante toda una serie compleja de determinaciones de su malestar, desconociéndose para esto la incidencia de factores socio afectivos, culturales, económicos, familiares y pedagógicos puestos "en juego" en los modos de expresión que éstos ponen de manifiesto a través de su desatención e hiperactividad en las aulas.

\section{La niñez y adolescencia "discapacitada mental"}

Asimismo, resulta preocupante observar en relación a esta problemática el significativo incremento de niños y jóvenes portando "certificados de discapacidad" por "trastornos mentales" de distinto tipo. Todo hace pensar que éstos han pasado a ser un dispositivo clave en estos procedimientos a los que con llamativa ligereza parecen apelar ahora no pocos profesionales capturados por estos discursos medicalizadores, y a los que se les vienen sumando en los últimos años, e impulsados por los mismos, algunas asociaciones de padres y familiares organizados en torno a los respectivos "trastornos mentales" con los que han sido "etiquetados" sus hijos como ADD-H, TGD, TEA, DEA, $\mathrm{RM}$, etc. Cuestión ésta que nos remite por su parte a considerar el uso de un nuevo término, el de la "biomedicalización", al

\footnotetext{
6 Teniendo particularmente en cuenta que, en estos tiempos tempranos de la vida, los sujetos están en pleno proceso de constitución de su subjetividad.
} 
que se esta apelando últimamente para explicar un nuevo giro dado en los últimos años en estos procesos de "medicalización de la sociedad".

\section{IV. ¿Por qué hoy hablamos de "biomedicalización”?}

De acuerdo a diferentes estudios que se vienen realizando sobre este tema y entre los que se destacan los desarrollos de Clarke y colegas (2010) este proceso al que se viene haciendo referencia, de transformación de la "medicalización" en "biomedicalización" fue posible por la confluencia de diferentes aspectos. En el caso de enfermedades y trastornos mentales ya conocidos como los mencionados ADD-H, TGD, TEA, etc., lo que las farmacéuticas hicieron fue expandir el mercado desarrollando nuevos mecanismos comunicacionales para que se internalice el "problema" como un "trastorno" subdiagnosticado y que puede ser controlado por fármacos. Para esto, la industria pasó de un modelo centrado en la "educación de los profesionales de la salud", en especial los médicos para que prescriban sus productos, a otro dirigido directamente a los consumidores.

Tratándose de niños, las campañas de comercialización se focalizaron en los padres y maestros. En países latinoamericanos, advierten las autoras mencionadas, se observa la utilización de campañas de concientización de enfermedades usando los medios masivos de difusión pero sin nombrar la medicación, y presentaciones en ámbitos educativos o en programas de radio o TV donde "expertos" en el tema "educan" a la audiencia para que sean capaces de identificar los síntomas.

Estos espacios de información son mantenidos inclusive por organizaciones gubernamentales que ofrecen a los usuarios a suscribirse para recibir noticias y actualizaciones sobre distinto tipo de "trastornos", "síndromes" o "deficiencias cognitivas" como el ADD-H, el TGD, el TEA y ahora también la re-editada "Dislexia" a la que ahora se la explica a partir de una supuesta deficiencia cromosomática. 
La industria farmacéutica brinda también apoyo financiero a asociaciones de padres y de pacientes para que difundan sus trastornos a través de distintos sitios en la red.

Inmersos en estas circunstancias, aquellos niños, niñas $y$ jóvenes que presentan modos de ser y estar en el mundo, de jugar, comunicarse y aprender "diferentes" a las expectativas normativas de una sociedad que parece tener estandarizados los patrones de lo que se considera "normal", aparecen signados por el fantasma del "fracaso escolar", y estrechamente ligado a éste, a modo de profecía, el de su exclusión "a futuro" en lo social y en lo económico. Se inicia entonces un proceso que, a partir de la estigmatización, potencia las dificultades para tomar conciencia de las posibilidades que supone la niñez, en tanto sujetos en pleno devenir, y por consiguiente de las estrategias a las que se pudiera apelar para promover un desarrollo más completo de las mismas, simplificándose a la vez las complejidades de la vida psíquica infantil.

Asimismo, determinados sentidos y modos de comprensión de un "problema" quedan relegados así a un plano secundario que habilita el pasaje de la descripción de "síntomas" a la determinación de supuestas "patologías".

\footnotetext{
7 "Normal" término que erróneamente se utiliza como sinónimo de "saludable" en la infancia.

8 Al respecto, resulta necesario advertir cómo el mencionado Manual -cuyo paradigma parece apoyarse en una concepción del ser humano como un mero soporte biológico de diversas funciones cognitivas desvinculadas en su constitución del resto del psiquismo y de toda referencia al otro, su historia y al medio- establece los criterios de "diagnóstico" al estipular la cantidad de indicadores conductuales que deben estar presentes para poder evaluar diverso tipo de "trastornos mentales", de modo tal que éstos, al aplicarse sobre niños, niñas y adolescentes, terminan basándose en una especie de anamnesis focalizada en aquellas funciones cognitivas "bajo sospecha", obtenida a partir de los datos provistos por los padres, maestros y cuidadores del niño, en base a escalas y sondeos, como el Cuestionario de Conners, el CHAT, etc. considerados como los principales instrumentos de "evaluación" para su detección e identificación, omitiéndose además, y en todo momento, considerar la fiabilidad científica de estos procedimientos y, por lo tanto, el sesgo que puede tener la información otorgada por los informantes.
}

Ahondando en estos temas, resulta necesario considerar que uno de los principales supuestos erróneos del que parte el DSM en este tipo de procedimientos, consiste en 
De este modo, el predominio del modelo médico biologicista-reeducador, en el cual la medicación, con frecuencia acompañada de "programas multidisciplinarios de adiestramiento conductual", aparece como "la solución" a un hipotético déficit orgánico portado desde el nacimiento, opera, como se anticipaba, a modo de "obturador" de toda relación e interrogación que habilite la posibilidad de poder "escuchar" al niño, niña u adolescente, invisibilizándose incluso, de esta manera, y en no pocas ocasiones, diferentes situaciones socio afectivas que le ocasionan sufrimiento psíquico?.

Atentos a estos aportes, ningún profesional de la salud debiera entonces quedarse tranquilo cuando se diagnostica algo supuestamente orgánico sin que ni siquiera existan test de laboratorios o estudios por imágenes, establecidos y confiables que los corroboren, y en su lugar, se proceda a reducir diversas problemáticas a observaciones impregnadas de posibles prejuicios, escalas de conducta muy poco objetivas, demasiado dependientes de preconceptos de los padres y de los profesionales intervinientes, tareas de ejecución y tests psicológicos de validez incierta, etcétera.

Por último, un asunto no menor que no podemos dejar de considerar en relación a lo que anticipábamos, es que este tipo de abordajes induce a las "adicciones" en la medida que la "solución" rápida que se les propone para todo tipo de dificultades y

considerar lo biológico como invariable punto de partida pues se lo entiende como centro emisor causal, sin estimar la posibilidad de la dirección inversa o recíproca.

9 Es por todas estas razones que consideramos que este tipo de intervenciones constituyen operaciones fuertemente desubjetivantes. Y esto no sólo para el niño, la niña o el adolescente que queda así "anulado como alguien que pueda decir algo acerca de lo que le pasa". En efecto, resulta necesario advertir además que este tipo de modelo también afecta a los mismos profesionales que, capturados por estos discursos medicalizadores, ejercen este tipo de prácticas que bien pueden calificarse de "tecnocráticas", en la medida que les restan posibilidades de escuchar, pensar, preguntarse e intentar entender los problemas ante los cuales se ven interpelados cotidianamente tanto desde la clínica como desde las aulas, y frente a los cuales, sólo parecen saber responder de manera "protocolizada", de acuerdo a las indicaciones provistas por el famoso Manual DSM, a quienes muchos refieren que hoy parece haberse transformado en una especie de "Biblia" en el campo de la Salud Mental. 
o malestares escolares, familiares o sociales parecen venir de la mano de alguna "pastillita mágica".

\section{A modo de cierre}

En función de lo expuesto, parece necesario que los profesionales que nos abocamos a la niñez y adolescencias tengamos presente que, por sobre todas las cosas, nuestra prioridad siempre y en todo momento, tanto desde el ámbito de la salud como desde lo social y la educación, debiera ser "escuchar" al niño u adolescente, cómo éste pueda expresarse, de modo de poder "entender" lo que le puede estar pasando y así "atenderlo" como se merece, respaldándonos para esto en toda la nueva legislación vigente a nivel nacional. De ahí la necesidad de conocerla y remitir a ella al revisar nuestra praxis.

Necesitamos romper con el discurso dominante que estructura una realidad naturalizada y ahistórica ${ }^{10}$. Lograr un profundo replanteo de nuestras miradas y discursos desde una praxis vinculada a las necesidades y sufrimientos de la población. Desde esta perspectiva se nos impone entonces una reflexión crítica que historice nuestro que-hacer, revirtiendo la cultura mercantilista que encierra, silencia y excluye a nuestros niños niñas y adolescentes, de modo de poder construir colectivamente dispositivos inclusivos, subjetivantes, que garanticen el derecho a vivir una infancia, una juventud, amparada por un mundo adulto que logre alojarla, contenerla, propiciarla. Derechos todos esenciales para la construcción de una ciudadanía plena.

\section{Bibliografía}

Benasayag \& Dueñas Comps. (2011) Invención de Enfermedades. Ed Noveduc. Buenos Aires.

\footnotetext{
10 No es la primera vez que la salud es usada como "Caballo de Troya" del poder económico y del poder represivo. Muchas clínicas y manicomios públicos y privados están allí para dar testimonio de ello. Parafraseando a Foucault, hoy deberíamos hablar de "Vigilar y Facturar".
} 
Dueñas, G.(2013). Niños en peligro. La escuela no es un hospital. Ed Noveduc. Buenos Aires.

Follari, R. (2007). La interdisciplina en la docencia"POLIS-Revista Latinoamericana.

Foucault, Michel (2002). Vigilar y Castigar. Ed. Buenos Aires.

Galende, E. y Lodieu, Nabergoi y Sopransi (2012) Equipos interdisciplinarios en Salud Mental Comunitaria Departamento de Salud Comunitaria. UNLa.Virtual / Universidad Nacional de Lanús

Iriart, C, e Iglesias Ríos (2012). "Biomedicalización e infancia: trastorno de déficit de atención e hiperactividad" en Interface. Comunicación, salud y Educación. Vol 16. Numero 43. Brasil, Oct/Diciembre

Menéndez E. (1985). Modelo médico hegemónico, crisis socio-económica y estrategias de acción en el sector salud. Cuadernos Médicos Sociales No 33, Buenos Aires.

Pelento, Marilú (2011) "Incidencia de los cambios culturales en la subjetividad de los niños", en Dueñas, G, Comp (2011). La patologización de la infancia: ¿Niños o síndromes?. Ed Noveduc. Buenos Aires.

Van Dijk, Teun A.(2005). Ideología y Análisis del Discurso Universitat Pompeu Fabra, Barcelona, España -Utopía y Praxis Latinoamericana / Afio 10. $\mathrm{N}^{\circ} 29$

- (2005) Política, Ideología y Discurso Quórum Académico- Vol. 2. Julio-diciembre 2005, • ISSN 1690- Universidad del Zulia 